\title{
Applicabilité du modèle cognitif des images schématiques dans l'enseignement-apprentissage de l'écrit au niveau universitaire
}

\author{
Applying Image Schemas to Teaching Writing \\ at the University Level
}

Joanna Kopeć

Université de Łódź, Pologne

Résumé : Les images schématiques sont des structures basiques qui émergent directement de notre expérience corporelle. Bien que dépourvues de contenu propositionnel, elles constituent directement le domaine source des métaphores des images schématiques (image schema metaphors) et participent indirectement à l'émergence des concepts plus spécifiques et plus élaborés. L'auteure examine l'image schématique du CHEMIN ainsi que ses modèles subsidiaires dans le cadre de la linguistique cognitive ef évalue leur applicabilité dans l'enseignement des langues étrangères. Les notions d'image schématique et de métaphore conceptuelle ne sont pas uniquement théoriques mais permettent de structurer un texte et un discours. L'auteure démontre comment elles peuvent être appliquées dans l'enseignement de l'écriture figurative qui inclut toutes les formes d'écriture et ne se limite pas à la poésie ou à la fiction.

Mots-clés: image schématique, métaphore conceptuelle, enseignement-apprentissage de l'écrit universitaire, didactique du français idiomatique.

Abstract: Image schemas are basic and skeletal structures directly emerging out of our bodily experience. Though deprived of propositional content, they directly constitute the source domain of image schema metaphors and indirectly participate in the emergence of more specific and elaborate concepts. The author discusses the PATH image schema along with its subsidiary schemas within the Cognitive Linguistics framework and assesses their applicability to foreign language teaching. Image schemas and conceptual metaphors are not only theoretical constructs but they have psychological reality and play a role in structuring texts and discourses. The author demonstrates how they can be applied in teaching figurative writing which, according to the cognitive view of figurative language and thought, includes all forms of writing and is not limited to poetry or fiction.

Keywords: image schema, conceptual metaphor, image schema metaphor, foreign language teaching, teaching and writing at the university level, idioms. 
Écrire est une façon de se connecter à autrui, à d'autres cultures, d'autres pays, d'autres océans. Être humain est un apprentissage, cela ne s'arrête jamais.

Joy Harjo

\section{Introduction}

L'enseignement-apprentissage de l'écrit au niveau avancé constitue l'une des préoccupations majeures des didacticiens depuis des décennies. Dès l'apparition des méthodes dites communicatives et plus tard actionnelles, cette capacité, qui de nos jours s'avère plus qu'indispensable, a été pendant longtemps mise à l'écart. II en découle que l'intérêt des apprenants pour l'écrit reste incontestablement moindre que celui porté à l'oral. II est clair cependant que l'écriture constitue un outil cognitif efficace permettant de structurer la pensée. Dans notre pratique de l'enseignementapprentissage de l'écrit, nous tentons de mettre l'accent sur la compréhension de la structure des langues, d'où l'intérêt que nous portons aux recherches en linguistique cognitive. Cet article, dont le but est de répondre aux besoins des apprenants du français et de l'espagnol langues étrangères du niveau avancé, a été conçu dans le cadre d'études effectuées à l'Institut d'Études Romanes de l'Université de Lodz. Après avoir lu, analysé et corrigé un corpus argumentatif de 350 textes en langue étrangère (le français) rédigés par des étudiants de troisième année de licence, nous avons pu y constater, qu'outre les problèmes auxquels on se heurte depuis longtemps (emploi douteux du lexique, cohésion textuelle déficiente), on observe également une très faible présence d'expressions métaphoriques. Nous avons donc tenté de répondre à la question suivante : comment aider les apprenants à acquérir ce langage figuratif et assurer sa rétention linguistique? Partant du principe que la linguistique cognitive accorde une grande importance à l'enseignement du langage figuratif, tout en soulignant la valeur communicative de ce dernier dans la communication interculturelle, nous voulons proposer une implication didactique reposant sur deux concepts fondamentaux de la LC, à savoir : les images schématiques (Johnson, 1987) et la métaphore conceptuelle (Lakoff et Johnson, 1980). Ces deux modèles théoriques nous permettent de mettre en valeur la base métaphorique et conceptuelle des expressions idiomatiques (Gibbs, 1994) qui, en plus d'être une source considérable de créativité lexicale, devraient occuper la place qu'elles méritent dans l'enseignementapprentissage des langues étrangères. Afin de contribuer à l'enseignement du langage métaphorique dans les cours de langues étrangères, nous avons établi, à partir des textes journalistiques, un corpus d'expressions idiomatiques provenant des images schématiques subsidiaires à celle du CHEMIN. Nous avons ensuite étudié la conceptualisation spatiale ainsi que les manifestations linguistiques en français et en espagnol de toutes les expressions du corpus, et avons élaboré une série d'activités lexicales et de production écrite. Nous avons choisi une étude comparative dans le but de faciliter aux étudiants l'acquisition des expressions métaphoriques qui, dans les deux langues romanes qu'ils apprennent, sont basées sur les mêmes images schématiques.

Le point de vue cognitif que nous avons adopté, nous autorise à supposer que l'incorporation de la compétence métaphorique (Littlemore, 2006) dans l'enseignement-apprentissage de l'écrit au niveau universitaire du français langue 
étrangère et de l'espagnol langues étrangères nous montrera dans quelle mesure la conscience de la valeur figurative des langues aidera les apprenants à contourner les difficultés sémantiques et leur permettra ainsi d'accéder au vrai sens (compréhension écrite) et ensuite de construire un vrai sens du texte (production écrite).

\title{
1. Les images schématiques : définition(s) et classification
}

Le concept d'image schématique, étroitement lié à ceux de langue figurative et de cognition incarnée (ang.: embodied cognition), est le socle de la Théorie de la Métaphore Conceptuelle. II constitue le niveau le plus schématique de la conceptualisation de notre expérience. La définition formulée par Johnson est celle qui a été citée par la majorité des linguistes et qui est la plus employée en linguistique cognitive :

Prenons d'abord une image schématique, qui est un modèle dynamique qui fonctionne un peu comme une structure abstraite d'image, et se connecte donc à une vaste gamme d'expériences différentes qui manifestent le même modèle récurrent (Cusimano 2008, pp. 77-80).

Il est important d'ajouter que la description des images schématiques ne se limite pas à un seul critère, celui de l'abstraction. Selon Claustner et Croft :

\begin{abstract}
Les images schématiques structurent notre expérience corporelle (Talmy, 1972, 1977, 1983), et elles structurent aussi notre expérience non-corporelle, à travers la métaphore (Lakoff, 1987, p. 453 ; Johnson, 1987, p. 29). Cette définition clarifie la description apparemment contradictoire des images schématiques qu'on rencontre parfois : or les images schématiques sont en un sens " abstraites » puisqu'elles sont schématiques, mais non " abstraites " dans un autre sens puisqu'elles sont dans le corps. (1999, p. 14)
\end{abstract}

Ces modèles cognitifs sont «incarnés» à travers nos expériences perceptives corporelles et puisqu'ils font intervenir notre perception sensorielle, nous pouvons insister sur leur aspect topologique, comme le fait Peña Cervel (cit. d'après Cusimano, 1999, p. 188) :

Les images schématiques sont des conceptualisations topologiques abstraites qui peuvent être utilisées pour donner une structure à une grande variété de domaines cognitifs.

Nous avons décidé d'élaborer un corpus d'expressions idiomatiques en français et en espagnol en nous basant sur le schéma conceptuel du CHEMIN car, à notre avis, c'est celui qu' on retrouve le plus souvent dans le discours. II va être exploité en classe de langue afin de faciliter aux étudiants l'acquisition du langage figuratif. L'image schématique du CHEMIN vient de notre expérience vitale des objets en mouvement (qui parfois sont forcés de se déplacer) et qui poursuivent leur route malgré les obstacles. Chaque chemin possède un point de départ et un but, comme nous pouvons l'observer sur le dessin suivant (Peña Cervel, 2012, p. 70) sur lequel figurent les principaux composants de cette image schématique. 
Entité en mouvement

Origine

Destination

Figure 1. Image schématique du CHEMIN

Quand nous disons: Jean a franchi le premier la ligne d'arrivée/Juan llegó el primero a la meta, nous nous référons à l'image schématique du CHEMIN. Jean serait l'entité en mouvement, le chemin correspondrait à l'itinéraire reliant le point de départ à la ligne d'arrivée qui est la destination (COURSE - CHEMIN). Les images schématiques nous aident donc à comprendre les exemples métaphoriques comme aller à la dérive/ir a la deriva où le modèle cognitif du CHEMIN nous permet de conceptualiser une situation soumise aux aléas de la vie (RELATION - VOYAGE CHEMIN).

Il est important de souligner qu'il existe différentes taxonomies d'images schématiques. Dans notre article, nous voudrions présenter la classification proposée par Peña Cervel (2012, p. 75) dans laquelle la chercheuse tente de pallier les principales déficiences des études antérieures, à savoir celles de Johnson (1987), Cienki (1997) ou Turner (1993). L'auteure distingue les images schématiques basiques et subsidiaires en prenant en considération la relation de dépendance que ces images entretiennent entre elles. L'image schématique du CHEMIN est considérée comme un modèle basique par rapport auquel les autres schémas jouent un rôle subsidiaire: c'est le cas de FORCE, PROCESSUS, PRÈS-LOIN, DEVANT-DERRIÈRE, VERTICALITÉ, CYCLE-SPIRALE.

\section{La description des images schématiques subsidiaires à celui du CHEMIN}

Nous voudrions à présent définir brièvement les schémas conceptuels subsidiaires au schéma basique du CHEMIN, ce qui nous permettra de mieux comprendre la façon dont ils sont structurés et d'observer les relations établies entre eux.

FORCE - la relation de ce schéma avec le CHEMIN est évidente. Dans la phrase : La fuerza del viento destruyó varios árboles/La force du vent a détruit de nombreux arbres, le vent se présente comme une force d'origine inconnue qui suit une trajectoire vers une destination, à savoir les arbres. À cause du pouvoir destructif de ladite force, ceux-ci se sont retrouvés gravement endommagés.

PROCESSUS - les processus peuvent être interprétés comme les étapes du chemin parcouru. El niño se transformó en un gran hombre/Le garçon est devenu un homme grand, l'exemple sert à illustrer ce schéma conceptuel de la manière suivante : le garçon constitue le point initial, les différentes phases de la vie du garçon sont les 
points intermédiaires du chemin et le fait de devenir un homme grand est lié à la destination.

PRÈS - LOIN - ce modèle expérientiel définit une autre orientation et se compose de deux ou plusieurs entités : le chemin et la distance entre lesdites entités (si la distance est grande nous parlons de l'image schématique LOIN et, si elle est petite, c'est l'image schématique PRÈS qui va être activée). Les expressions suivantes permettent d'exemplifier cette image schématique d'orientation : María estuvo cerca de la muerte/Marie a frôlé la mort.

DEVANT - DERRIÈRE - voilà une autre image schématique qui émane de notre expérience. Au moyen de ce modèle, nous pouvons nous référer à un mouvement en avant ou en arrière ainsi qu'à la localisation d'une entité déterminée. La source ou le point de départ peuvent être considérés comme DERRIÈRE, la destination ou l'arrivée constituent DEVANT (AVANT). Une série de points intermédiaires entre la source et la destination font progresser le "trajecteur" de la source vers le but. Les exemples illustratifs de cette image schématique seraient les suivants : Juan siempre se sienta en la parte trasera de la clase/Jean s'assoit toujours au fond de la classe.

VERTICALITÉ - l'image schématique associée à l'orientation HAUT/BAS, peut être interprétée comme un chemin à l'orientation verticale. À titre d'exemple, nous pouvons mentionner : Juan subió al quinto piso/Jean est monté au cinquième étage où nous remarquons une entité, Jean, qui effectue un mouvement ascendant et arrive à la destination, au cinquième étage. Un autre exemple, cette fois métaphorique, serait Elena estaba en el séptimo cielo/Hélène était au septième ciel. Dans ce cas, Hélène se trouve dans un endroit doté de connotations positives en raison de son orientation élevée, à savoir, dans le ciel.

CYCLE - SPIRALE - ce modèle provient de notre expérience vécue, des changements cycliques de la nature au fil des jours ou des saisons (le printemps, l'été, l'automne, l'hiver; le jour et la nuit, etc.). Le cycle peut être interprété comme un chemin circulaire vers lequel convergent le point de départ et la destination. Mentionnons, en qualité d'exemple, la phrase suivante : La luna nueva es el comienzo de un nuevo ciclo de fases lunares/La nouvelle lune c'est le début du nouveau cycle de phases lunaires.

Ce bref parcours à travers les images schématiques subsidiaires à celui du CHEMIN nous a permis de mieux comprendre la nature incorporée de ces modèles cognitifs et d'observer de plus près leur grand potentiel structurant. Ils fournissent le domaine source aux métaphores d'orientation ou aux métaphores des images schématiques, et constituent ainsi des modèles à caractère applicable en didactique des langues étrangères.

\section{La métaphore conceptuelle}

La présence du langage métaphorique dans notre vie quotidienne est beaucoup plus fréquente que nous ne le pensons. Le locuteur natif ne se rend même pas compte du fait qu'il s'exprime en termes métaphoriques, c'est-à-dire qu'il aborde un concept à l'aide d'un autre, avec lequel, en principe, il n'entretient aucune relation. Rappelons que les expériences physiques récurrentes nous permettent de structurer des notions abstraites sous forme d'images schématiques, et de nous exprimer par des métaphores (LA VIE - UN VOYAGE - UN CHEMIN). Celles-ci sont organisées de façon 
systématique et cohérente, par exemple : traverser la vie/pasar por la vida, arriver à faire qch/llegar a hacer algo, nous ne pouvons pas faire marche arrière maintenant/no podemos dar marcha atrás ahora. La question est donc de savoir si l'apprenant d'une langue étrangère peut arriver à comprendre ce langage figuratif sans passer par la fastidieuse étape de mémorisation des listes d'expressions idiomatiques. Comme l'explique Boers (2000, 2004), la réflexion sur les métaphores facilite la compréhension du langage figuratif, éclaire l'origine polysémique de beaucoup de mots et favorise ainsi l'apprentissage du vocabulaire.

La métaphore conceptuelle est un phénomène de cognition défini d'après les termes employés par Lakoff et Johnson comme un ensemble de correspondances entre deux domaines conceptuels, le domaine source et le domaine cible. II est important de distinguer la métaphore conceptuelle de la métaphore linguistique. Cette dernière concerne les mots ou les expressions linguistiques issus du domaine source de la métaphore conceptuelle, généralement plus concret ou cognitivement et intersubjectivement plus accessible du point de vue conceptuel (Kopeć, Przybysz, 2020 , p. 140). Les métaphores linguistiques peuvent varier d'une langue à l'autre, bien qu'elles soient des exemplifications linguistiques de la même métaphore conceptuelle. Ainsi, les expressions employées métaphoriquement : aller à la dérive et ir a la deriva proviennent de la même image schématique du CHEMIN, plus particulièrement de la DÉVIATION et ne diffèrent pas linguistiquement. Ici, les métaphores conceptuelles sont identiques dans les deux langues, c'est-à-dire : DIFFICULTÉS SONT DES OBSTACLES. En revanche, être au pied du mur, estar entre la espada y la pared, représentent une image schématique subsidiaire de la COMPULSION, et en comparant ces deux expressions métaphoriques, nous remarquons des différences dans la décomposition scénique de la même situation. À la lumière de ce qui vient d'être dit, il nous paraît nécessaire de souligner que :

\begin{abstract}
la recherche des métaphores conceptuelles peut aider les apprenants, en général, à comprendre les textes, et en particulier, à reconnaître des effets rhétoriques; de même la reconnaissance de domaines métaphoriques sources permet d'identifier les champs d'expérience culturellement et historiquement significatifs ou représentatifs (stéréotypiques), notamment de manière contrastive : en espagnol, le domaine religieux, en français celui de la nourriture, sont à la base de nombreuses expressions idiomatiques (Bayle 2009).
\end{abstract}

L'image schématique du CHEMIN constitue une base cohérente pour l'apprentissage des expressions figées liées à la métaphore conceptuelle AMOUR EST UN VOYAGE. C'est une conceptualisation du domaine abstrait de l'amour, grâce au domaine plus concret du voyage, à l'aide d'une série de correspondances (mappings) structurées et caractéristiques pour la langue et la culture en question. Observons, par exemple, comment les locuteurs natifs parlent de l'amour : tôt ou tard toute relation se retrouve dans une impasse 1/tarde o temprano toda relación llega a un punto muerto, nous sommes à la croisée des chemins²/estamos en la encrucijada, nous ne pouvons pas faire marche arrière3/ no podemos dar marcha atrás, ce chemin

\footnotetext{
1 https://theraforma.com [12/02/2021].

2 https://linternaute.fr/expression/langue-française [12/02/2021].

3 https://expressio.fr [12/02/2021].
} 
ne nous mène nulle part 4 / este camino no nos lleva a ninguna parte, notre relation n'avance pas/nuestra relación no está avanzando, c'est un grand pas en avant pour notre couple5/es un gran paso adelante para nuestra pareja, etc. Les expressions linguistiques en italique rendent évidentes les correspondances métaphoriques cidessous:

DOMAINE CIBLE : AMOUR

amants

relation amoureuse

projet de couple

difficultés vécues

choix à effectuer

évolution de la relation
DOMAINE SOURCE : VOYAGE

voyageurs

véhicule

destination du voyage

obstacles à surmonter

choix du chemin

distance parcourve

Figure 2. Les correspondances métaphoriques (Kövecses, 2010, p. 9)

L'une des caractéristiques principales de la métaphore conceptuelle est qu'elle constitue un cas spécifique d'une métaphore plus générale, ce qui prouve sa structure hiérarchique. Par exemple, LES ACTIVITÉS AVEC OBJECTIF ET DE LONGUE DURÉE sont conceptualisées, d'une manière générale, comme un VOYAGE, plus exactement le voyage métaphorique d'un couple vers une destination bien déterminée (Soriano, 2012).

Toutes ces considérations nous amènent à faire le lien entre la métaphore conceptuelle et la métaphore de l'image schématique. Comme l'affirme Kövecses:

Image-schema metaphors are based on "skeletal" image-schemas, such as the path-schema, the force-schema, the contact schema, and the like. They are skeletal in the sense that these source domains do not map rich knowledge onto the target. (2010, p. 326)

Le modèle cognitif de l'image schématique en tant que concept de niveau d'expérience physique fondamental, ne possède pas de domaines sources riches ni complexes, néanmoins participe à la création de la pensée abstraite. Son importance réside dans sa capacité explicative, qui lui permet d'aborder la structure des métaphores des images schématiques (image schema metaphors), p. ex. : AMOUR EST UN VOYAGE, SIMILARITÉ EST LA PROXIMITÉ.

\section{Quelques pistes didactiques}

Comme nous l'avons vu dans les sections antérieures, les images schématiques et la métaphore conceptuelle se présentent comme des outils cognitifs fondamentaux dans la déduction du sens et la structuration de la pensée. Ces modèles peuvent être appliqués dans le cadre de cours de langue, en tant que moyens de conscientisation sur les formes très variées de penser et de concevoir la réalité, ainsi que sur les

${ }^{4}$ https://question-ortographe.fr [12/02/2021].

5 https://www.cosmopolitan.fr [12/02/2021]. 
différences culturelles qui imprègnent la signification d'une expression. II esł probable qu'un apprenant du français ou de l'espagnol à qui on présente la métaphore conceptuelle d'une expression qu'il ne connaît pas, se rende compte du fait que la même métaphore existe dans sa langue maternelle ou dans une autre langue romane qu'il est en train d'apprendre. Boers (2000) attire l'attention sur la nature métaphorique du langage par le biais d'activités qui aident à associer l'expression métaphorique à sa motivation, ce qui contribue à créer la conscience métaphorique et facilite l'apprentissage des expressions. Danesi (1988) recommande de développer chez les apprenants d'une langue étrangère une compétence qu'il nomme «la compétence métaphorique » et qu'il définit comme les connaissances portant sur la codification et la représentation des concepts abstraits à l'aide des métaphores.

\subsection{L'enseignement du langage figuratif en trois dimensions (Gómez 2013, p. 92)}

Dans cette section, nous proposons une série d'applications didactiques ${ }^{6}$ qui visent à faciliter l'apprentissage des expressions métaphoriques correspondant au schéma conceptuel lié au domaine spatial, c'est-à-dire : à l'image schématique du CHEMIN. Ces considérations didactiques sont présentées à travers trois dimensions: métalinguistique, conceptuelle et linguistique. La dimension métalinguistique concerne la compréhension de la notion de métaphore. La dimension conceptuelle s'articule sur l'enseignement des structures conceptuelles liées à l'image schématique du CHEMIN ainsi qu'à ses images subsidiaires. Finalement, la dimension linguistique dont le rôle est de mettre en relation les schémas conceptuels mentionnés auparavant avec les éléments linguistiques qui leur correspondent.

\subsubsection{Dimension métalinguistique : comprendre le concept de métaphore}

L'enseignement du langage figuratif devrait être précédé par un travail sur la compréhension de la notion de métaphore. Pour ce faire, il nous semble efficace de proposer une activité qui permettra aux étudiants de s'exprimer avec des métaphores (Gascón, 2011, p. 152).

\footnotetext{
${ }^{6}$ Les contenus des activités sont des idées originales de l'auteure de l'article.
} 
Tableau 1. Découvrir les métaphores

a) Qu'est-ce qu'une métaphore ? Formulez une définition de la métaphore et donnez quelques exemples, en français, en espagnol et dans votre langue maternelle.

b) Souvent, nous utilisons des mots simples pour faire référence à des idées abstraites et compliquées (comme, par exemple, à des événements importants de notre vie). Les expressions proposées ci-dessus sont des exemples en français et en espagnol de la métaphore L'AMOUR EST UN VOYAGE/AMOR ES UN VIAJE :

- Nous sommes à la croisée des chemins/Estamos en la encrucijada

- Je pense que cette relation ne nous mène nulle part/Creo que esta relación no nos lleva a ninguna parte

- Cette relation est une impasse/Esta relación llega a un punto muerto.

- Nous ne pouvons pas faire demi-tour maintenant/Ahora no podemos dar marcha atrás.

Cette étape de conscientisation sera suivie par l'enseignement conceptuel du langage métaphorique afin de faire découvrir aux étudiants les métaphores de l'image schématique du CHEMIN.

\subsubsection{Dimension conceptuelle : développer la compétence conceptuelle}

Nous considérons que l'image schématique du CHEMIN que nous avons présentée au préalable, devrait, en raison de sa systématicité et de sa fréquence en langues française et espagnole, occuper une place considérable dans l'enseignement du langage figuratif.

\section{Enseignement conceptuel}

a) L'activité ci-dessous est basée sur un corpus d'expressions linguistiques métaphoriques provenant de textes journalistiques. Elle consiste à faire réaliser aux étudiants le statut métaphorique des expressions ainsi qu'à rendre explicite le contexte théorique: les métaphores conceptuelles et linguistiques ainsi que les domaines source et cible. II s'agit également de développer chez les apprenants la capacité de savoir comment convertir les schémas de pensée issus de différents domaines conceptuels en structures linguistiques adéquates. 
Tableau 2. Le contexte théorique

À partir des expressions linguistiques métaphoriques soulignées ${ }^{7}$, trouvez le domaine source et le domaine cible des métaphores des images schématiques. Répondez aussi à la question suivante : quelle(s) image(s) schématique(s) repérez-vous dans le domaine source?

- Olga Tokarczuk est recompensée pour « une imagination narrative qui, avec une passion encyclopédique, symbolise le dépassement des frontières comme forme de vie $»$.

- Peter Handke est distingué quant à lui pour une œuvre qui "forte d'ingénuité linguistique, a exploré la périphérie et la singularité de l'expérience humaine ».

- En réalité, on ne fait que la moitié du chemin, ces mesures ne règlent en rien leur parcours du combattant pour se loger, notamment...

- La situation dans le Finistère montre une circulation du virus en augmentation.

- Elena estaba en el cielo tras saber que había aprobado todo.

Le but de cette activité est d'amener les apprenants à reconnaître les métaphores suivantes: CONNU EST CENTRAL, INCONNU EST PÉRIPHÉRIQUE, PROGRÈS EST UN MOUVEMENT EN AVANT, PROGRÈS EST UNE DISTANCE PARCOURUE, HAUT EST LE BONHEUR, ensuite à nommer les images schématiques des domaines sources respectives (CENTRE-PÉRIPHÉRIE, CHEMIN et VERTICALITÉ).

b) Le travail sur des textes académiques constitue une occasion d'étudier la conceptualisation spatiale en présentant l'activité mentale en termes de mouvement physique : recueillir des idées, ramasser des idées, passer à un nouveau point, revenir au point précédent, aboutir à une connaissance approfondie, apporter des informations précieuses, arriver à distinguer, des certitudes sont balayées.

c) Une autre possibilité est de faire apparaître les mécanismes de la métaphore conceptuelle à travers l'analyse des textes littéraires. Les étudiants travaillent alors en groupe ou individuellement, essaient de reconnaître les expressions et de trouver les métaphores conceptuelles qui leur correspondent. Grâce à cette démarche, ils peuvent employer, de manière consciente, le langage figuratif, qui s'appuie sur la conceptualisation métaphorique.

\subsubsection{Dimension linguistique : enseigner le langage métaphorique}

La principale difficulté de l'enseignement de la dimension linguistique du langage métaphorique est due au fait que pour exprimer les schémas conceptuels partagés par les langues connues des apprenants, il faut employer les structures différentes. En outre, il ne faut pas oublier que les manifestations linguistiques de la métaphore

\footnotetext{
7 Les exemples viennent du corpus créé à partir des textes journalistiques (tirés essentiellement de L'Express et de El País).
} 
conceptuelle représentent des niveaux de conventionalité, d'extension (une phrase, un discours) et de fixation (collocations, expressions figées) très variés. Comme l'affirme Sacristán (2009), des activités de traduction peuvent également être utiles afin de mettre en lumière des similarités et des différences, qui sont liées au langage métaphorique, entre la langue maternelle des apprenants et la/les langue(s) cible(s). De notre point de vue, l'étude comparative constitue une stratégie fondamentale dans l'apprentissage des expressions métaphoriques. La citation ci-dessous confirme, à notre avis, l'importance d'une telle approche (Jorge 1992, p. 129) :

L'expérience, les valeurs humaines, contiennent des traits universaux. La forme qui les anime peut se présenter différemment d'une langue à l'autre, mais la valeur sémantique et les concepts sous-jacents à ces formes peuvent rapprocher les langues.

Il nous semble donc opportun de proposer aux apprenants une activité d'écriture créative dans laquelle ces derniers doivent rédiger deux textes narratifs sur l'amour, conceptualisé soit comme un voyage soit comme un échange économique. Nous pensons que l'emploi de la métaphore conceptuelle représente une source de créativité dans les productions écrites et permet aux apprenants de développer leurs compétences de rédaction.

\section{Conclusion}

Cet article avait pour but de susciter la réflexion sur les possibilités du développement de la compétence métaphorique au niveau universitaire. II nous a permis de mettre en valeur la présence des schémas conceptuels particulièrement récurrents, CHEMIN, FORCE, PROCESSUS, PRÈS-LOIN, DEVANT-DERRIÈRE, VERTICALITÉ, CYCLE-SPIRALE, communs à la langue française et à la langue espagnole. Nous avons essayé de répondre à la question: par quels moyens nous pouvons rendre l'enseignementapprentissage du langage figuratif plus efficace? Notre raisonnement s'est essentiellement appuyé sur deux concepts fondamentaux de la LC : la métaphore conceptuelle et les images schématiques, car nous considérons qu'un enseignementapprentissage des langues étrangères basé sur ces concepts garantit la compréhension des métaphores linguistiques conventionnelles et nonconventionnelles. L'utilité pratique des images schématiques dans l'acquisition des langues étrangères nous semble très pertinente dans la mesure où elles constituent les modèles du plus haut niveau de schématisation et permettent de trouver le domaine source de plusieurs métaphores conceptuelles. II convient d'ajouter que le développement de la compétence métaphorique se produit généralement à partir du niveau intermédiaire et doit être corrélé à la maîtrise de la langue. Les apprenants qui ont atteint un niveau satisfaisant de compétence linguistique doivent être capables de comprendre et de produire un langage métaphorique. La compétence métaphorique peut donc être considérée comme un facteur déterminant de la compétence linguistique des apprenants en langues étrangères. II reste à savoir comment mesurer la pertinence des modèles cognitifs des images schématiques et quelle est la meilleure façon de les insérer dans les séquences didactiques. Nos réflexions précèdent l'étape de la mise en pratique des propositions didactiques pour l'enseignement du langage figuratif, à la suite de laquelle, nous nous attendons à des résultats convaincants et innovants. 


\section{Bibliographie}

ACHARD BAYLE, G. (2009). La linguistique cognitive et ses applications. Recherches en Didactique des Langues et des Cultures : Les Cahiers de l'Acedle, 2, pp. 39-68.

BOERS, F. (2000). Metaphor awareness and vocabulary retention. Applied Linguistics, 4, pp. 553-571.

Clausner, T. C. \& CROFt, W. (1999). Domains and image schemas. Cognitive Linguistics, 10, pp. 1-31.

Cusimano, C. (2008). La polysémie. Essai de sémantique générale. Paris: L'Harmattan.

DANESI, M. (1986). The Role of Metaphor in Second Language Pedagogy. Rassegna Italiana di Linguistica Applicata, 18, pp. 1-10.

GOMEZ, L. (2013). L'expression métaphorique de l'événement émotionnel en français (L1) et en espagnol (L1/L2) : Les images schéma haut/bas et dedans/dehors. Analyse descriptive et proposition didactique. Recherches en Didactique des Langues et des Cultures: Les Cahiers de l'A cedle, 10, pp. 81-102.

HIJAZO-GASCÓN, A. (2011). Las metáforas conceptuales como estrategias comunicativas y de aprendizaje: Una aplicación didáctica de la lingüística cognitiva. Hispania, 1, pp. 142-154.

IBARRETXE-Antuñano, I. \& VenezUela, J. (ed.). (2012). Lingüística Cognitiva. Barcelona: Anthropos.

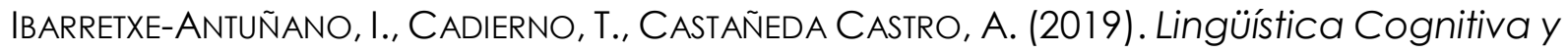
español LE/L2. London, New York: Routledge.

JOHNSON, M. (1987). The body in the mind: The bodily basis of meaning, imagination, and reason. Chicago: University of Chicago Press.

JORGE, G. (1992). Les expressions idiomatiques correspondantes: analyse comparative. Terminologie \& Traduction, 2-3, pp. 127-134.

KOPEĆ, J. \& PRZYBYSZ, A. (2020). Le diable se cache dans les mots: une analyse linguistique et cognitive du discours populiste suivie de son application didactique. In SZKOPIŃSKI, Ł. \& WOCH, A. (ed.), Populismo y Propaganda : entre el presente y el pasado. Łódź : Wydawnictwo Uniwersytetu Łódzkiego, pp. 137-148.

KÖVECSES, Z. (2010). Metaphor. A practical introduction. Oxford : Oxford University Press.

LAKOFF, G., \& JOHNSON, M. (1980). Metaphors We Live By. Chicago: University of Chicago Press.

LITTLEMORE, J. \& LOW, G. (2006). Metaphoric competence and communicative language ability. Applied Linguistics, 2, pp. 268-294.

PEÑA CERVEL, M. S. (1999). Subsidiary relationships between image schemas: an approach to the force schema. Journal of English Linguistics, 1, pp. 187-207.

Peña Cervel, M.S. (2012). Los esquemas de imagen. In Ibarretxe-Antuñano, I. \& Valenzuela, J., Lingüística Cognitiva. Barcelona: Anthropos, pp. 69-96.

SORIANO, C. (2012). La metáfora conceptual. In Ibarretxe-Antuñano, I. \& Valenzuela, J. Lingüística Cognitiva. Barcelona: Anthropos, pp. 97-121. 
VELASCO SACRISTÁN, M. (2009). A translation approach to metaphor teaching in the LSP classroom : simple exercises from a Business English syllabus. Ibérica, 17, pp. 8398. 